\title{
Comparison of Three Different Techniques of Inverted Internal Limiting Membrane Flap in Treatment of Large Idiopathic Full-Thickness Macular Hole
}

\author{
Fariba Ghassemi' \\ Hassan Khojasteh' \\ Alireza Khodabande' \\ Lauren A Dalvin (D) ${ }^{2}$ \\ Mehdi Mazloumi (D) ${ }^{1,3}$ \\ Hamid Riazi-Esfahani $\mathbb{I D}^{\prime}$ \\ Masoud Mirghorbani' \\ 'Eye Research Center (ERC), Farabi Eye \\ Hospital, Tehran University of Medical \\ Sciences, Tehran, Iran; ${ }^{2}$ Department of \\ Ophthalmology, Mayo Clinic, Rochester, \\ MN, USA; ${ }^{3}$ Wills Eye Hospital, Thomas \\ Jefferson University, Philadelphia, \\ PA, USA
}

This article was published in the following Dove Press journal: Clinical Ophthalmology

Purpose: To evaluate and compare three different techniques of inverted internal limiting membrane (ILM) flap in the treatment of large idiopathic full-thickness macular hole.

Methods: In a comparative interventional case series, 72 eyes from 72 patients with large $(>400 \mu \mathrm{m})$ full-thickness macular hole were randomly enrolled into three different groups: group A - hemicircular ILM peel with temporally hinged inverted flap; group B - circular ILM peel with temporally hinged inverted flap; and group C - circular ILM peel with superior inverted flap. Best-corrected visual acuity (BCVA), anatomical closure rate, and ellipsoid zone (EZ) or external limiting membrane (ELM) defects were evaluated preoperatively, at week 1, and months 1,3 and 6 after surgery.

Results: There were 24 eyes in group A, 23 in group B, and 25 in group C. In all three groups, larger diameter macular hole was associated with worse preoperative visual acuity $(\mathrm{r}=0.625, \mathrm{P}<0.001)$. Mean BCVA improved significantly in all three groups 6 months after surgery $(0.91 \mathrm{vs} 0.55, \mathrm{p}<0.001) .6$ months after surgery, mean BCVA improved from 0.91 $\operatorname{logMAR}$ to $0.52 \pm 0.06$ in group A, 0.90 to $0.53 \pm 0.06$ in group B, and 0.91 to $0.55 \pm 0.11$ in group C. In group A vs. B vs. C, improvement of BCVA was $0.380 \pm 0.04$ vs. $0.383 \pm 0.04$ vs. $0.368 \pm 0.11 \log \mathrm{MAR}$, with no statistically significant difference between groups $(\mathrm{P}=0.660)$. The rate of successful hole closure was $87.5 \%$ vs. $91.3 \%$ vs. $100 \%$. Although the closure rate was $100 \%$ in Group C (circular ILM peel with superiorly hinged inverted flap), this difference was not statistically significant $(\mathrm{P}=0.115)$.

Conclusion: ILM peel with an inverted flap is a highly effective procedure for the treatment of large, full-thickness macular hole. Different flap techniques have comparable results, indicating that the technique can be chosen based on surgeon preference.

Keywords: internal limiting membrane peel, inverted flap, macular hole, pars plana vitrectomy

\section{Introduction}

Idiopathic macular hole $(\mathrm{MH})$ is a vitreoretinal disorder with a considerable impact on the quality of life, which affects approximately 8.7 eyes per 100,000 per year. ${ }^{1,2}$ Since the introduction of vitrectomy for macular hole treatment by Kelly and Wendel in 1991, numerous strategies have been investigated to improve visual acuity and anatomic outcomes, considering the underlying mechanism of disease. ${ }^{3}$ It has been proposed that the internal limiting membrane (ILM) plays a key role in the formation
Correspondence: Hassan Khojasteh Eye Research Center (ERC), Farabi Eye Hospital, Qazvin Square, PO Box: 14155 7I46, Tehran, Iran

Tel +982155421113

Fax +982155419095

Email khojaste_hasan@yahoo.com 
of macular hole through the exertion of tangential vitreomacular traction forces on the inner retinal layers, which could also be transmitted to the photoreceptor layer via Muller cells. ${ }^{4}$ Thus, ILM peel during vitrectomy has become an important component of macular hole surgery to achieve sustained visual and anatomic success. ${ }^{5}$ However, U-shaped closure, the most favorable type of closure with the best functional results, occurs in only $45 \%$ of cases. In $19-39 \%$ of cases, the result is flat macular hole margins with bare retinal pigment epithelium (W-type closure), flat-closed macular hole, or flat-open macular hole. ${ }^{6}$ The inverted ILM flap technique was first introduced in 2010 by Michalewska et al and showed superiority compared to traditional ILM peeling technique in terms of both closure rate and visual outcomes. $^{7}$

Recently, some concerns have been evoked regarding the possible complications associated with ILM peeling, including damage to Muller cells, formation of paracentral retinal holes, and dissociated optic nerve fiber layer (DONFL). ${ }^{8-10}$ Michalewska et al tried to minimize these iatrogenic traumas while maintaining good surgical outcomes by peeling off the ILM only from the temporal side of the fovea. ${ }^{7}$ Casini et al investigated a modification to the inverted ILM flap technique, using the fluid-air exchange in place of surgical manipulation to invert the remaining attached ILM over the macular hole, and found similar success compared to the Michaelewska technique. ${ }^{11}$ Flap displacement, however, remains a concern in the inverted ILM flap technique. Thus, we hypothesized that the orientation of the inverting flap (horizontal from temporal to nasal versus vertical from superior to inferior) could influence surgical outcomes.

Herein, we introduce a modification to the inverted ILM flap technique with circular ILM peeling and inferiorly inverted flap and compare this technique with previously reported approaches. We discuss visual and anatomic success in hemicircular ILM peel with temporally hinged inverted flap versus circular ILM peel with temporally hinged inverted flap versus circular ILM peel with superior inverted flap.

\section{Methods}

This study was implemented in accordance with the tenets of the declaration of Helsinki. The study protocol was approved by the local ethics review committee of Tehran University of Medical Sciences, and all participants provided written informed consent prior to inclusion.

\section{Study Participants}

In a prospective, interventional, comparative study, we enrolled 72 phakic eyes from 72 patients suffering from full-thickness MH with a minimum diameter exceeding 400 $\mu \mathrm{m}$. Patients with any history of other retinal pathology, including diabetic retinopathy, high myopia, and agerelated macular degeneration, were excluded. All patients underwent a comprehensive ophthalmic evaluation, including best-corrected visual acuity (BCVA), slit-lamp examination, and dilated funduscopy. The diagnosis of $\mathrm{MH}$ was confirmed by spectral-domain optical coherence tomography (SD-OCT, SPECTRALIS ${ }^{\circledR}$, Heidelberg Engineering, Germany). A raster imaging protocol consisting of 31 horizontal scans was obtained, which covered a $7 \times 9 \mathrm{~mm}^{2}$ area centered on the fovea. Size of $\mathrm{MH}$ was defined as a horizontal diameter at the narrowest point according to the International Vitreomacular Traction Study (IVTS) group classification of macular hole. ${ }^{12}$

Patients were randomized into three treatment groups based on block randomization technique: group A - hemicircular ILM peel with temporally hinged inverted flap; group B - circular ILM peel with temporally hinged inverted flap; and group C - circular ILM peel with superior inverted flap.

\section{Surgical Technique}

One surgeon (HK) performed all operations. All cases underwent 23-gauge pars plana vitrectomy with phacoemulsification and IOL implantation. ILM was stained by Brilliant Blue dye. In group A, the ILM was peeled off ( $\geq 2$ disc diameters) just from the temporal side of fovea and was then totally inverted to cover the hole (Figure 1). In groups B and C, the ILM was peeled off 360 degrees ( $\geq 2$ disc diameters) with minimal adhesion to the edges of the MH. In group B, the flap was inverted from temporal side to nasal side, while in group $\mathrm{C}$, the flap was inverted from superior to inferior (Figures 2 and 3) (Video 1).

Inversion of the created flap was done by passive suction during air-fluid exchange without direct touch or additional manipulation. After complete drying, the vitreous cavity was filled with $20 \%$ SF6. Patients were advised to maintain prone position for at least 3 days. Patients were examined at 1 week and 1, 3, and 6 months postoperatively. At each visit, patients underwent complete ophthalmologic examination including BCVA measurement, slit-lamp examination, indirect ophthalmoscopy, and SD-OCT. Postoperative anatomical success was 


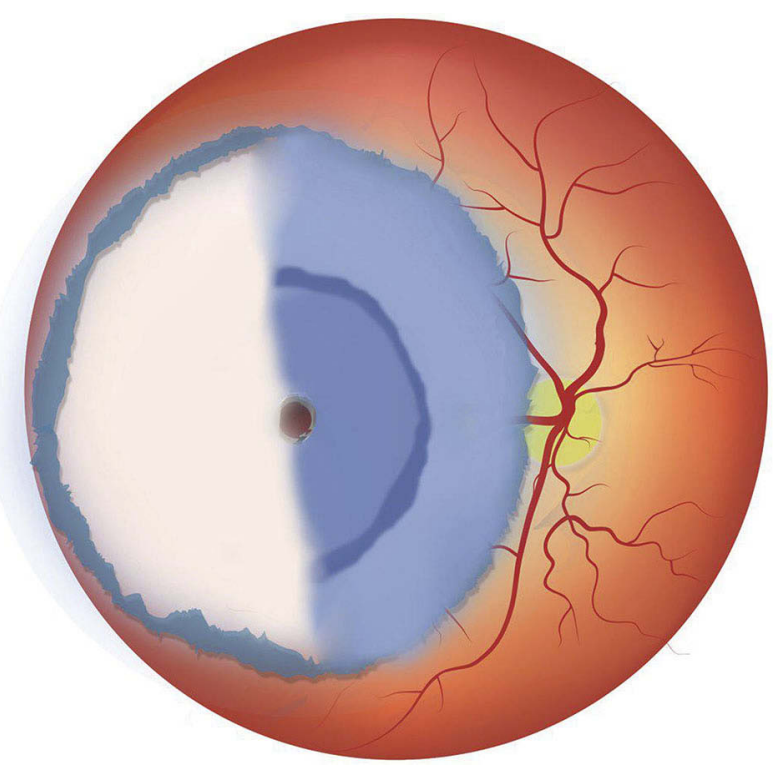

Figure I Schematic image of surgical technique for group A: ILM is peeled off from the temporal side of the fovea inverting to the nasal side. Final configuration of ILM is shown.

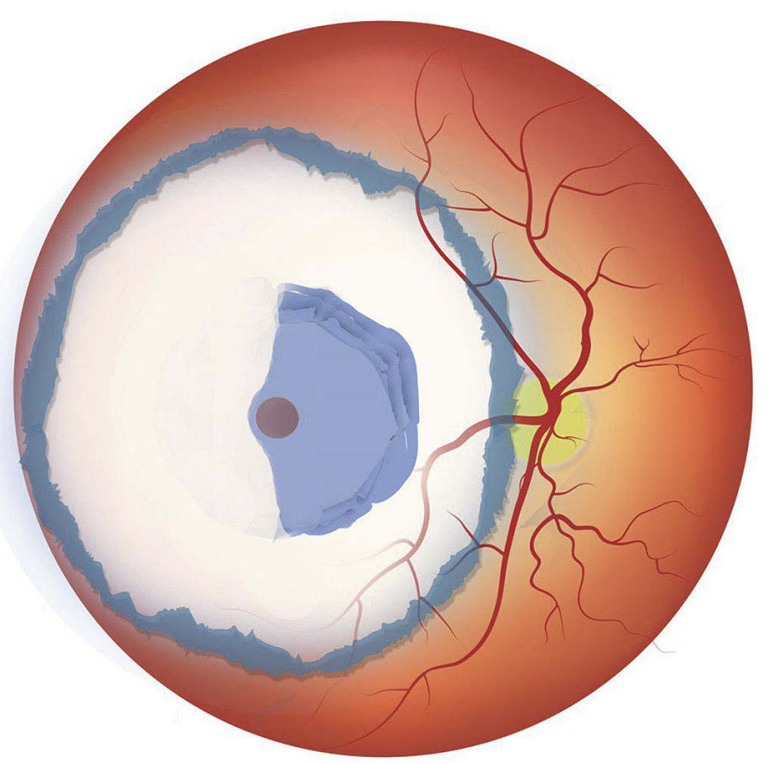

Figure 2 Schematic image of surgical technique for group B: ILM peeled off in a circular manner with minimal adhesion to the fovea, inverting horizontally from temporal to the nasal side. Final configuration of ILM is shown.

defined as $\mathrm{U}$ or V-shaped closure according to crosssectional morphology on SD-OCT.

\section{Statistical Analysis}

Data were analyzed using SPSS software (version 18 for Windows; SPSS, Inc., Chicago, IL, USA). Continuous variables are expressed as mean \pm Standard error of mean. Comparisons between the three groups were

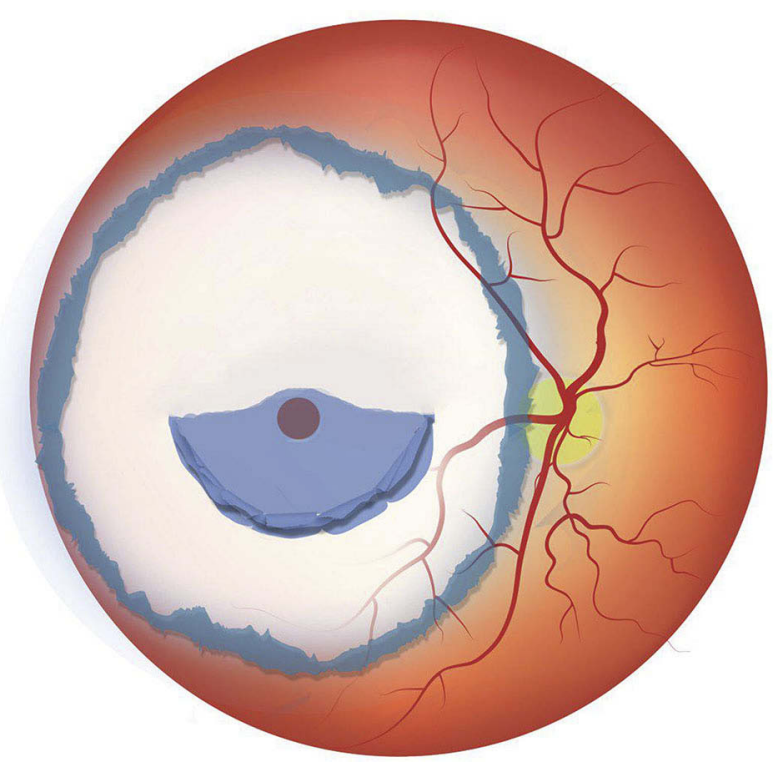

Figure 3 Schematic image of surgical technique for group C: ILM is peeled off in a circular manner with minimal adhesion to the fovea inverting vertically from superior to the inferior side. Final configuration of ILM is shown.

performed using the ANOVA test. Categorical variables were compared using the Fisher's exact test and Camer's $\mathrm{V}$ test. Binominal logistic regression was performed to assess the influence of preoperative BCVA, size of the $\mathrm{MH}$, age, sex, and type of surgical technique on the anatomic success rate of the surgery. $P \leq 0.05$ was considered statistically significant.

\section{Results}

The study included 72 eyes from 72 patients. There were 24 eyes randomized to group A (hemicircular ILM peel with nasally hinged flap), 23 to group B (circular ILM peel with nasally hinged flap), and 25 to group C (circular ILM peel with superior-inverted flap). Table 1 summarizes the baseline characteristics in each group. There were no significant differences in age, sex, baseline BCVA, or size of the $\mathrm{MH}$ between groups.

\section{Functional Results}

In all groups, mean BCVA improved significantly 6 months after surgery $(p<0.001)$. In group A, mean BCVA improved from $0.90 \pm 0.04 \log M A R$ to $0.85 \pm 0.04,0.65 \pm 0.05$, and 0.52 $\pm 0.06 \log \mathrm{MAR}$ at 1,3 , and 6 months after surgery, respectively. In group B, mean BCVA improved from $0.91 \pm 0.04$ to $0.84 \pm 0.06,0.66 \pm 0.03$, and $0.53 \pm 0.06 \log$ MAR at 1,3 , and 6 months after surgery, respectively. In group C, mean BCVA improved from $0.92 \pm 0.04$ to $0.86 \pm 0.03,0.66 \pm 0.04$, and $0.55 \pm 0.11 \log M A R$ at 1,3 , and 6 months after surgery, 
Table I Baseline Characteristics of the Patients in Three Groups

\begin{tabular}{|l|l|l|l|l|}
\hline & Group A n=24 & Group B n=23 & Group C n=25 & P-value \\
\hline Age, years & $67.04 \pm 0.62$ & $67.30 \pm 0.73$ & $67.28 \pm 0.71$ & 0.588 \\
Preoperative BCVA ${ }^{\text {a }}$ Log MAR & $0.90 \pm 0.04$ & $0.91 \pm 0.04$ & $0.92 \pm 0.04$ & 0.994 \\
Size of Macular Hole $(\mu \mathrm{m})$ & $552.63 \pm 21.21$ & $534.78 \pm 15.88$ & $548.40 \pm 20.64$ & 0.931 \\
Male/Female ratio & $10 / 14$ & $10 / 13$ & $9 / 16$ & 0.90 \\
\hline
\end{tabular}

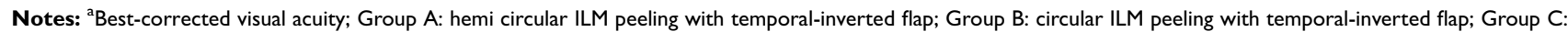
circular ILM peeling with superior inverted flap.

respectively. There was no statistically significant difference in final postoperative visual acuity between the three groups $(P=0.66)$. In all three groups, worse preoperative visual acuity correlated with larger $\mathrm{MH}$ diameter $(\mathrm{r}=0.63$, $P<0.001)$.

\section{Anatomical Results}

In this study, we defined successful anatomic closure as a V-shaped or U-shaped closure. Successful closure was noted in $87.5 \%$ (21/24) of cases in group A, 91.3\% (21/23) of cases in group B, and $100 \%(25 / 25)$ of cases in group C 1 week after primary surgery. Among patients with failed closure, three cases underwent a second operation within the first month. In group A, 2 cases underwent reoperation; one patient was treated with replacement of nasally hinged flap but the other patient was treated with an extension of ILM peeling as avulsion of the previous flap had been occurred. In group B, one case underwent reoperation with an extension of ILM peeling due to the previous flap avulsion. All three cases got closure 1 week after the reoperation. Two other patients refused to being reoperated and did not continue their follow up.

At 6 months after surgery, complete ellipsoid zone (EZ) regeneration was found in $52.4 \%$ of successfully closed cases in group A. Complete EZ regeneration was seen in $47.6 \%$ and $56.0 \%$ in groups B and C, respectively. There was no significant difference in likelihood of complete EZ regeneration between the three groups $(P=0.87)$.

OCT features of complete external limiting membrane (ELM) recovery were noted in $71.4 \%$ of successfully closed cases in group A at 6 months follow up. ELM recovery was noted in $66.6 \%$ and $72.0 \%$ in groups $\mathrm{B}$ and $\mathrm{C}$, respectively. There was no significant difference between the three groups $(P=0.89)$. Table 2 shows details of the postoperative visual and anatomic outcomes of patients in each group. Improvement of BCVA was not significantly different between groups $(P=0.66)$. Although the successful closure rate was $100 \%$ in group $C$ (circular ILM peel with inferiorly hinged flap), this difference was not statistically significant $(P=0.115)$. Binominal logistic regression analysis showed no significant relationship between surgical approach to ILM, age, sex, or size of the MH and the anatomic success rate of the surgery. The only variable which showed a significant effect was preoperative BCVA, with worse preoperative BCVA resulting in lower rate of successful MH closure $(P \leq 0.001)$.

\section{Patient One}

A 59-year-old lady presented with a large full-thickness MH in her left eye. The minimum size of the MH was $553 \mu \mathrm{m}$, and the basal diameter was $1100 \mu \mathrm{m}$ (Figure 4A). Her initial visual acuity was 20/100. Pars plana vitrectomy with hemicircular ILM peeling with nasally hinged flap (the technique used for group A) was performed, and the MH was closed 1 week postoperatively. One month after surgery, visual acuity improved to 20/50. An area of hyperreflective tissue on the inner retinal surface and nasal to fovea was seen on OCT, which corresponded to the location of the inverted ILM flap (Figure 4B). At 3 months, visual acuity improved to 20/40 with improvement in EZ irregularities. Besides outer retinal regeneration, some degree of inner retinal dimpling at the temporal side was observed at 3 months after surgery

Table 2 Anatomical and Visual Outcomes of the Patients in Different Groups 6 Months After Surgery

\begin{tabular}{|l|l|l|l|}
\hline & Closed/Not-Closed & Postoperative BCVA ${ }^{a}$ Log MAR & BCVA Improvement LogMAR \\
\hline Group A, $n=24$ & $21 / 3(87,5 \%)$ & $0.525 \pm 0.058$ & $0.380 \pm 0.04$ \\
Group B, $n=23$ & $21 / 2(91.3 \%)$ & $0.526 \pm 0.063$ & $0.383 \pm 0.04$ \\
Group C, $n=25$ & $25 / 0(100 \%)$ & $0.552 \pm 0.109$ & $0.368 \pm 0.11$ \\
\hline
\end{tabular}

Note: ${ }^{a}$ Best-corrected visual acuity. 

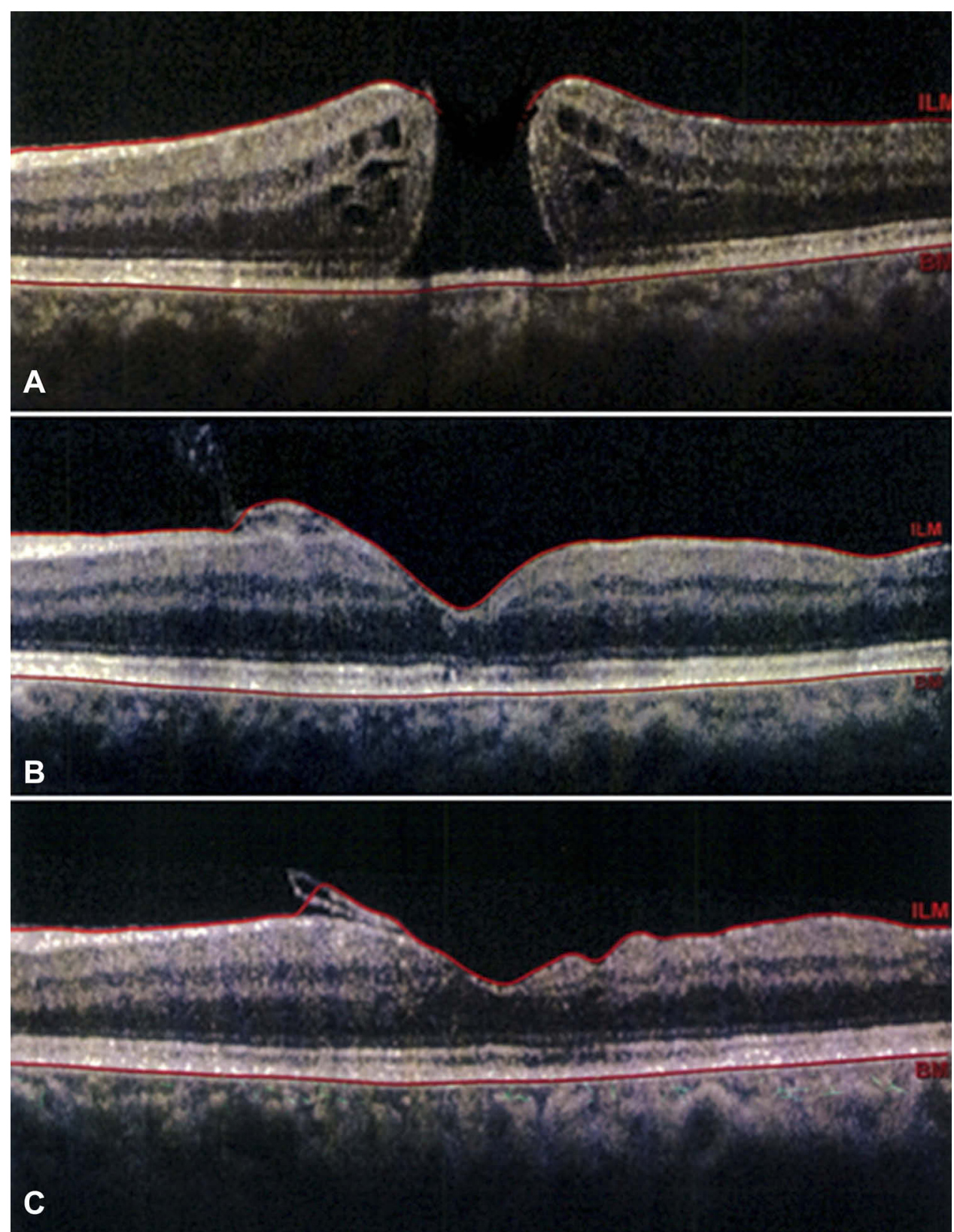

Figure 4 Case I. (A) Preoperative OCT shows a large full-thickness macular hole. (B) I month postoperatively, the inverted flap is visualized as a hyperreflective tissue nasal to the fovea. (C) OCT 3 months after surgery shows some degree of atrophic change in the inner retinal surface limited to the temporal side of fovea.

(Figure 4C). After 6 months of follow up, visual acuity improved to $20 / 30$.

\section{Patient Two}

A 65-year-old man presented with a large full-thickness MH with a minimal diameter of $744 \mu \mathrm{m}$ and basal diameter of $1337 \mu \mathrm{m}$ (Figure 5A). The initial visual acuity was 20/400. Pars plana vitrectomy with circular ILM peeling and superior inverted flap (the technique used for group C) was performed. The $\mathrm{MH}$ was closed 1 week after surgery. Visual acuity improved to 20/200 at
1 month postoperatively. OCT showed closed MH with two hyporeflective spaces, created by the inverted flap (Figure 5B). At 3 months after surgery, these spaces were filled with regenerated tissue with improved foveal contour (Figure 5C). Visual acuity was 20/63. Six months after surgery, visual acuity improved to 20/40, and a small defect in the ellipsoid zone with atrophic inner retinal changes was seen on OCT. Although the peeling was circular and involved both the temporal and nasal sides of fovea, inner retinal dimpling was most prominent at the temporal side (Figure 5D). 

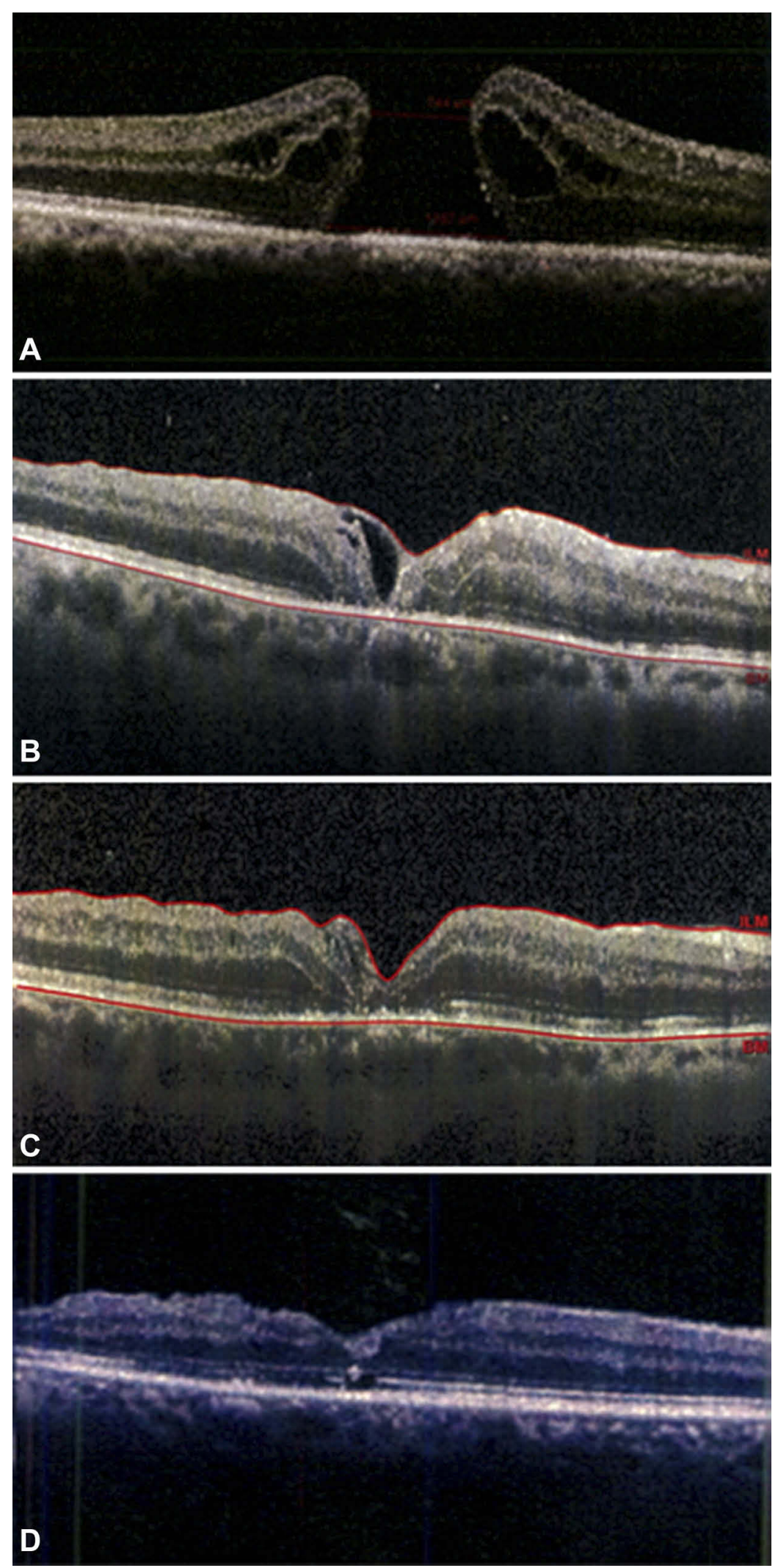

Figure 5 Case 2. (A) Preoperative OCT shows a large full-thickness macular hole. (B) I month postoperatively, closed macular hole is shown with two hyperreflective spaces created by an inverted flap. (C) These spaces were filled with regenerative tissue 3 months after surgery. (D) 6 months postoperatively, OCT shows regenerated outer retina with a small defect in the ellipsoid zone. Atrophic changes in the inner retinal surface are most prominently seen at the temporal side of the fovea. 


\section{Discussion}

Despite the proven insignificant difference in either costs or quality of life scores between MH surgery with or without ILM peeling, the first has gained so much popularity among vitreoretinal surgeons for treatment of full-thickness $\mathrm{MH}$ in the past decade. ${ }^{13}$ The pragmatic support for this approach arises from numerous clinical trials, which have supported the role of ILM peeling to achieve better anatomical and visual outcomes. ${ }^{14}$ This technique, however, suffers from a high failure rate, especially for large MHs. The inverted flap technique was first described by Michalewska et al in 2010 to improve visual and anatomic success. In this technique, during circumferential peeling, the ILM was not removed completely from the retina but was left attached to the edges of the MH. To cover the MH, the ILM remnant was then inverted upside-down. The authors hypothesized that the inverted ILM could act as a scaffold for glial cells to proliferate, enhancing closure of the MH. In addition, the ILM might serve as a barrier, disabling the entrance of fluid from the vitreous cavity into the hole. Satisfactory results expanded the use of this technique to myopic $\mathrm{MH}$, traumatic $\mathrm{MH}$, repeat $\mathrm{MH}$ surgery, and optic disc maculopathy. ${ }^{75-18}$ Yamashita et al compared conventional ILM peeling and inverted flap technique in both medium-large $(400-550 \mu \mathrm{m})$ and extra-large $(>550 \mu \mathrm{m}) \mathrm{MHs}$. They reported the closure rate of $95 \%$ and $100 \%$ in medium-large MHs by conventional and inverted flap techniques, respectively, and $88 \%$ and $100 \%$ in extra-large MHs although the difference between the two techniques was not significant. ${ }^{19}$

Although the inverted flap technique showed promising results in idiopathic $\mathrm{MH}$, especially for large holes, concerns arose regarding possible complications and technical difficulties. Edema of the arcuate nerve fiber layer has been noted, which is followed by the formation of small dimples in the retinal nerve fiber layer, called dissociated optic nerve fiber layer (DONFL), documented with OCT and autofluorescence imaging. ${ }^{20}$ Additionally, spontaneous stripping of the inverted ILM flap during fluid-air exchange has been reported in 14\% of cases. ${ }^{7}$ Although iatrogenic effects on the RNFL can disappear 1 to 3 months after surgery with no negative impact on visual acuity, ${ }^{20}$ Michalewska et al in another study introduced the temporal inverted ILM flap technique to minimize iatrogenic trauma associated with ILM peeling by decreasing the area of peeled ILM. In this technique, the ILM was peeled off at the temporal side of the $\mathrm{MH}$ in an area of about two disc diameters. During peeling, the ILM was not removed completely from the retina but instead was left attached to the temporal edge of the $\mathrm{MH}$ and was then inverted and coaxed over the $\mathrm{MH}$ until adequate coverage was achieved. Michalewska et al compared the modified technique with the classic inverted ILM flap technique and found no significant difference in visual or anatomic outcomes with the exception of more DONFL in the latter group. ${ }^{21}$ The authors mentioned that the temporal inverted flap would provide a scaffold for $\mathrm{MH}$ wall gliosis at the top of the $\mathrm{MH}$ space without creating a possible obstacle to the bottom of the MH compared to the presence of small-trimmed ILM flaps in the original technique. Furthermore, this type of large flap with a wider connection to the retina might not detach spontaneously and might not easily flip during fluid-air exchange. Although the minimalistic approach to ILM peeling would theoretically minimize iatrogenic trauma to the RNFL and decrease the risk of iatrogenic paracentral holes during surgery, the main reason for increasing popularity seems to be the ease of technique.

In the present study, we introduced another minimalistic approach, superior inverted flap technique (group C), and compared this technique with the previously mentioned techniques in the literature, ${ }^{21}$ hemicircular ILM peeling with temporal-inverted flap (group A) and circular ILM peeling with temporal-inverted flap (group B). In group C, we created an inverted flap from superior side of the fovea, flipping it downward to the inferior side. We found a successful $\mathrm{MH}$ closure rate of $100 \%$ in this group. However, this result was not statistically significant, probably due to our small sample size. We suspect that the main reason for high success rate with superior inverted flap is the creation of a more stable flap with more powerful inward tangential forces that enhance hole closure. By creating a vertical flap, gravitational forces can also assist in successful MH closure. Larger studies might reveal significant differences in visual and anatomic success compared with other surgical techniques.

After performing logistic regression, we interestingly found that the type of surgical technique in our study would not affect the postoperative anatomical success rate. Compatible with our findings, Bae et al have recently shown that increasing the area of ILM peeling might not increase the likelihood of MH closure. ${ }^{5}$ Indeed, the only measures in their study which showed a significant decrease were asymmetric elongation of fovea and degree of patient's metamorphopsia. We believe that restricting the area of peeling to one side of the macular area would not sacrifice the success rate of closure. In fact, the most important step during surgery is to release the tangential traction forces of ILM exerted on the foveal edges thereby allowing the glial Muller cells to start bridging at the bottom of the hole. In this way, the most favorable technique would be the 
one that is the easiest approach in one surgeon's hand. In this study, we also found no significant difference regarding VA improvement among our groups.

The main limitation of our study is the small number of patients, which limits the ability to detect small differences and raises the possibility of type II errors. Another limitation is the short time of follow up in each group.

In conclusion, we found that ILM inverted flap is a highly effective procedure in treating large fullthickness MHs. Larger studies with longer follow-up are needed to determine whether different flap orientations affect visual and anatomic success.

\section{Disclosure}

The authors declare that they do not have any conflict of interest and they do not have any financial interest to disclose.

\section{References}

1. McCannel CA, Ensminger JL, Diehl NN, Hodge DN. Populationbased incidence of macular holes. Ophthalmology. 2009;116 (7):1366-1369. doi:10.1016/j.ophtha.2009.01.052

2. Duan HT, Chen S, Wang YX, Kong JH, Dong M. Visual function and vision-related quality of life after vitrectomy for idiopathic macular hole: a 12mo follow-up study. Int J Ophthalmol. 2015;8(4):764-769. doi:10.3980/j.issn.2222-3959.2015.04.22

3. Kelly NE, Wendel RT. Vitreous surgery for idiopathic macular holes. Results of a pilot study. Arch Ophthalmol. 1991;109(5):654-659. doi:10.1001/archopht.1991.01080050068031

4. Ezra E. Idiopathic full thickness macular hole: natural history and pathogenesis. Br J Ophthalmol. 2001;85(1):102-108. doi:10.1136/ bjo.85.1.102

5. Bae K, Kang SW, Kim JH, Kim SJ, Kim JM, Yoon JM. Extent of internal limiting membrane peeling and its impact on macular hole surgery outcomes: a randomized trial. Am J Ophthalmol. 2016;169:179-188. doi:10.1016/j.ajo.2016.06.041

6. Kang SW, Ahn K, Ham DI. Types of macular hole closure and their clinical implications. $B r \quad J$ Ophthalmol. 2003;87(8):1015-1019. doi:10.1136/bjo.87.8.1015

7. Michalewska Z, Michalewski J, Adelman RA, Nawrocki J. Inverted internal limiting membrane flap technique for large macular holes. Ophthalmology. 2010;117(10):2018-2025. doi:10.1016/j.ophtha.2010. 02.011

8. Lim JW, Kim HK, Cho DY. Macular function and ultrastructure of the internal limiting membrane removed during surgery for idiopathic epiretinal membrane. Clin Exp Ophthalmol. 2011;39(1):9-14. doi:10.1111/j.1442-9071.2010.02377.x

Clinical Ophthalmology

\section{Publish your work in this journal}

Clinical Ophthalmology is an international, peer-reviewed journal covering all subspecialties within ophthalmology. Key topics include: Optometry; Visual science; Pharmacology and drug therapy in eye diseases; Basic Sciences; Primary and Secondary eye care; Patient Safety and Quality of Care Improvements. This journal is indexed on PubMed

Submit your manuscript here: https://www.dovepress.com/clinical-ophthalmology-journal
9. Steven P, Laqua H, Wong D, Hoerauf H. Secondary paracentral retinal holes following internal limiting membrane removal. $\mathrm{Br}$ J Ophthalmol. 2006;90(3):293-295. doi:10.1136/bjo.2005.078188

10. Ito Y, Terasaki H, Takahashi A, Yamakoshi T, Kondo M, Nakamura M. Dissociated optic nerve fiber layer appearance after internal limiting membrane peeling for idiopathic macular holes. Ophthalmology. 2005;112(8):1415-1420. doi:10.1016/j.ophtha.2005.02.023

11. Casini G, Mura M, Figus M, et al. Inverted internal limiting membrane flap technique for macular hole surgery without extra manipulation of the flap. Retina. 2017;37:2138-2144. doi:10.1097/ IAE.0000000000001470

12. Duker JS, Kaiser PK, Binder S, et al. The International Vitreomacular Traction Study Group classification of vitreomacular adhesion, traction, and macular hole. Ophthalmology. 2013;120(12):2611-2619. doi:10.1016/j.ophtha.2013.07.042

13. Ternent L, Vale L, Boachie C, et al. Cost-effectiveness of internal limiting membrane peeling versus no peeling for patients with an idiopathic full-thickness macular hole: results from a randomised controlled trial. Br J Ophthalmol. 2012;96(3):438-443. doi:10.1136/ bjophthalmol-2011-300402

14. Spiteri Cornish K, Lois N, Scott N, et al. Vitrectomy with internal limiting membrane (ILM) peeling versus vitrectomy with no peeling for idiopathic full-thickness macular hole (FTMH). Cochrane Database Syst Rev. 2013;6:CD009306.

15. Kuriyama S, Hayashi H, Jingami Y, Kuramoto N, Akita J, Matsumoto M. Efficacy of inverted internal limiting membrane flap technique for the treatment of macular hole in high myopia. Am J Ophthalmol. 2013;156 (1):125-131 e121. doi:10.1016/j.ajo.2013.02.014

16. Michalewska Z, Michalewski J, Dulczewska-Cichecka K, Nawrocki J. Inverted internal limiting membrane flap technique for surgical repair of myopic macular holes. Retina. 2014;34(4):664-669. doi:10.1097/IAE.0000000000000042

17. Abou Shousha MA. Inverted internal limiting membrane flap for large traumatic macular holes. Medicine (Baltimore). 2016;95(3): e2523. doi:10.1097/MD.0000000000002523

18. Mohammed OA, Pai A. Inverted autologous internal limiting membrane for management of optic disc pit with macular detachment. Middle East Afr J Ophthalmol. 2013;20(4):357-359. doi:10.4103/ 0974-9233.120008

19. Yamashita T, Sakamoto T, Terasaki H, et al. Best surgical technique and outcomes for large macular holes: retrospective multicentre study in Japan. Acta Ophthalmol (Copenh). 2018;96(8):e904-e910. doi:10.1111/ aos. 13795

20. Clark A, Balducci N, Pichi F, et al. Swelling of the arcuate nerve fiber layer after internal limiting membrane peeling. Retina (Philadelphia, Pa.). 2012;32(8):1608-1613. doi:10.1097/IAE.0b013e3182437e86

21. Michalewska Z, Michalewski J, Dulczewska-Cichecka K, Adelman RA, Nawrocki J. Temporal inverted internal limiting membrane flap technique versus classic inverted internal limiting membrane flap technique: a comparative study. Retina (Philadelphia, Pa.). 2015;35(9):1844-1850. doi:10.1097/IAE.0000000000000555
Central and CAS, and is the official journal of The Society of Clinical Ophthalmology (SCO). The manuscript management system is completely online and includes a very quick and fair peer-review system, which is all easy to use. Visit http://www.dovepress.com/ testimonials.php to read real quotes from published authors. 